\title{
Energy Cost Optimization in Microgrids Using Model Predictive Control and Mixed Integer Linear Programming
}

\author{
Rama K. Bonthu*, Ricardo P. Aguilera, Ha Pham, Manh Duong Phung and Quang P. Ha \\ University of Technology Sydney, Ultimo, NSW 2007, Australia. \\ \{RamaKishore.Bonthu; Ricardo.Aguilera; NgocHa.Pham; manhduong.phung; Quang.Ha\}@uts.edu.au
}

\begin{abstract}
This paper presents a model predictive control (MPC) approach based on the mixed integer linear programming (MILP) to develop an optimal power management strategy (PMS) for minimizing the electricity bill of commercial buildings in a domestic on-grid system. The optimal PMS is first formulated as a MILP-MPC with time-varying constraints. The constraints are then linearized at each sampling time so that a receding horizon principle can be used to determine the control input applied to the plant and update the model. The time-varying efficiency of power electronic converters is evaluated for each time interval and assumed to be persistent for the prediction time horizon. The numerical results show that the proposed MILP-MPC strategy with variable efficiency is effective in utilizing photovoltaic power generation to save the cost on electricity for buildings.
\end{abstract}

\section{NOMENCLATURE}

\section{Acronyms}

BESS battery energy storage system

MILP mixed integer linear programming

MPC model predictive control

PMS power management strategy

PV photovoltaic

RBC rule-based control

SoC state of charge

\section{Constants}

$N \quad$ number of discrete time intervals

$N_{p} \quad$ prediction horizon

$P_{\max }^{b u y} \quad$ maximum allowable buying power from grid [kW]

$P_{\max }^{c h} \quad$ maximum allowable charging power $[\mathrm{kW}]$

$P_{\min }^{c h} \quad$ minimum allowable charging power $[\mathrm{kW}]$

$P^{\text {dis }}$

$\max _{P^{\text {dis }}}$

$P_{\text {min }}$

SoC $C^{\max }$

SoC $C^{\text {min }}$ maximum allowable discharging power $[\mathrm{kW}]$ minimum allowable discharging power $[\mathrm{kW}]$ maximum allowable selling power to grid $[\mathrm{kW}]$ upper limit of state-of-charge [kWh]

$T_{s} \quad$ discrete time interval duration

\section{Decision variables}

$\delta^{b} \quad$ binary variable for charging/discharging power from/to BESS

$\delta^{g} \quad$ binary variable for buying/selling power from/to the grid

$P^{b u y} \quad$ power bought from the grid $[\mathrm{kW}]$

$P^{\text {ch }} \quad$ power exchanged with BESS during charging $[\mathrm{kW}]$

$P^{\text {dis }} \quad$ power exchanged with BESS during discharging $[\mathrm{kW}]$

$P^{\text {sell }} \quad$ power sold to the grid $[\mathrm{kW}]$

Indices

$\wedge \quad$ predicted value $j \quad$ prediction horizon intervals

$k \quad$ discrete time intervals

\section{Parameters}

$\boldsymbol{u}^{\text {opt }} \quad$ optimal input sequence

$\eta^{\text {ch }} \quad$ efficiency of BESS connected converter during charging

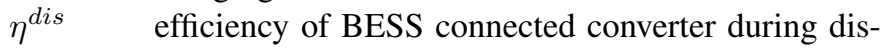
charging

$\eta^{p v} \quad$ efficiency of PV system connected converter

$c^{f} \quad$ feed-in tariff [AUD/kWh]

$c^{\text {tou }} \quad$ time-of-usage energy price [AUD/kWh]

$P^{l} \quad$ load power demand [kW]

$P^{p v} \quad$ power generation from $\mathrm{PV}$ system $[\mathrm{kW}]$

$\mathrm{SoC} \quad$ state-of-charge [kWh]

\section{INTRODUCTION}

Over the decades, the utilization of sustainable power sources has received a remarkable interest because of increasing electricity demand, the need to decrease $\mathrm{CO}_{2}$ emissions and the decay of fossil fuel resources. In this situation, photovoltaic (PV) and wind power generation appear as the most promising renewable resources. Prevalently, PV generation is rapidly developing in the residential energy sector for which it is particularly relevant. In present times, governments are assuring the PV development by giving incentive policies which detail lessened costs. Due to that factor, grid-tied PV systems are intended to uphold popularity for the following decade. As a result of the irregular nature of solar radiation, power management strategy (PMS) becomes complex. An imbalance of dynamic power demand and the PV generation is a major challenge. As of late, the battery energy storage system (BESS) has been used in combination with a solar system to address this concern. Consequently, along with the utility grid, modern home power system also comprises BESS and PV system [1]. On the contrary, the energy management capability and effectiveness limit the integration of grid-connected BESS.

Home PMS can control and supervise the power flow from/to the grid for matching the power consumption to that of the PV generation and also to reduce the overall cost of electrical power delivered to the residential systems. A BESS device introduces an extra investment cost and also has operating constraints. Moreover, power electronics converters are used as an interface of a home energy system and, thus, the consideration of the dynamic efficiency of these power converters should account for the real-time power conversion losses. These together with the dynamic energy pricing model 
for energy consumption from the grid and feed-in tariff will involve high nonlinearities, yielding a complicated optimization problem, for which the main objective is to find the charging/discharging schedule of BESS which minimizes the electricity bill.

Different methodologies for microgrid energy management have been introduced in the literature. Most of the studies related to the cost optimization techniques concentrate on a PMS between distributed generation, BESS and connected loads for minimizing the electricity cost, improving generation efficiency, saving energy and stabilizing the power system [2]. Because of the unpredictability of the microgrid optimization and extensive financial advantages that could come about because of enhanced solutions, extensive consideration is being committed to the improvement of better optimization algorithms and suitable modeling frameworks [3], [4]. Most of the proposed solution techniques incorporate mathematical programming [5], heuristics [6] and priority rules [7]. The authors in [8] proposed energy management solutions by applying a model predictive control (MPC) approach that considering fixed power conversion loss which is making the problem unrealistic. In [9], MPC methodology is applied to tackle the dynamic economic dispatch problem, aimed at minimizing the generation cost over a finite time horizon. Many researchers centered the long-term objectives on the efficient utilization of renewable energy within microgrids, e.g., the designing of BESS schedule to store the electricity locally generated from renewable sources and reuse it during peak load demand periods. Furthermore, all works are comprised of linearities and solved with implicit MPC to achieve control objectives without updating the plant model.

It is observed in the existing literature that the formulation of saving energy bills into a mixed integer linear programming (MILP) optimization problem with time-varying constraints has not been addressed. As MILP mostly uses the branch-andbound algorithm which gives the global optimum solution with minimum computational time, this approach can be applied to more complex microgrids. As such, we propose here to use a MILP-MPC for dealing with dynamic control and state constraints while satisfying performance specifications. This proposed control strategy provides advantages in terms of feedback control technique to predict the future response of the plant over a finite horizon, incorporates constraints explicitly, easy to formulate as a constrained optimization problem, closed-loop stability and inherent robustness [10]. The objective function and constraints are formulated into a finite-time optimal control problem. At each sampling period, a set of system states are updated, the optimal control problem is solved online, and the controller time horizon recedes by another step [11]. To the best of our knowledge, there are no current models including power electronic converters with consideration of dynamic efficiencies due to intermittent nature of renewable generation and variable load demand.

This paper also compares the results obtained from the proposed MILP-MPC with those from a rule-based control (RBC) algorithm. The RBC algorithm determines all the

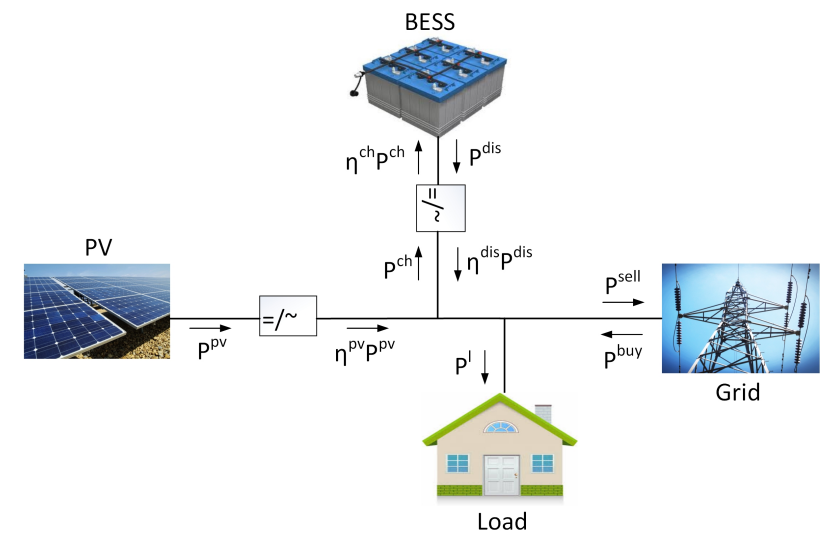

Fig. 1: A case study of microgrid.

control inputs based on a series of rules of 'if \& then conditions' which are associated with numerical values that need to be chosen. Our contributions are: $(i)$ the formulation of the building energy cost for a microgrid into a multiobjective optimization problem subject to variable constraints; $(i i)$ the development of a MILP-MPC scheme to deal with timevarying constraints involved in the problem.

\section{System Description, Modeling And Constraints}

In this work, the focus is on minimizing the electricity bill of a typical residential commercial building, using an integrated PV system and BESS with consideration of the dynamic efficiency of power converters as depicted in Fig. 1. Here, the target is to minimize the energy cost for a week by optimally managing the flow from the PV power and power exchanged with BESS during charging and discharging based on a dynamic electricity pricing model. For this case, a slotted time approach is used, whereby a week is divided into $N$ discrete time intervals and each interval with a duration of $T_{s}$ (e.g., $N=336$ for $T_{s}=30$ minutes).

\section{A. Storage Dynamics}

In the power management of microgrids, the state-of-charge (SoC) of BESS, plays an important role. For any sampling time $k$, its dynamics are described by [12]:

$$
S o C_{k+1}=S o C_{k}+T_{s} \eta_{k}^{c h} P_{k}^{c h}-T_{s} P_{k}^{d i s},
$$

A good range should be considered for the charging/discharging operation to improve the life of BESS. Such a constraint is expressed as:

$$
S o C^{\min } \leq S o C_{k} \leq S o C^{\max } .
$$

The BESS is restricted with the maximum allowable amount of power flowing in/out during charging/discharging, respectively. Therefore, the system must satisfy the following constraints:

$$
\begin{gathered}
\delta_{k}^{b} P_{\min }^{d i s} \leq P_{k}^{d i s} \leq \delta_{k}^{b} P_{\max }^{d i s} \\
\left(1-\delta_{k}^{b}\right) P_{\min }^{c h} \leq P_{k}^{c h} \leq\left(1-\delta_{k}^{b}\right) P_{\max }^{c h}
\end{gathered}
$$


According to the limitations on BESS power during charging/discharging, a binary variable, $\delta^{b}$, is considered, as per:

$$
\delta_{k}^{b}= \begin{cases}0 & \text { for charging } \\ 1 & \text { for discharging }\end{cases}
$$

\section{B. Grid interactions}

Here we consider a grid-tied system, which facilitates the power selling and buying from the utility grid. According to the standard approach discussed in [13], the building energy system must hold the following inequalities to sell and buy energy from the grid:

$$
\begin{gathered}
0 \leq P_{k}^{\text {buy }} \leq \delta_{k}^{g} P_{\text {max }}^{\text {buy }}, \\
0 \leq P_{k}^{\text {sell }} \leq\left(1-\delta_{k}^{g}\right) P_{\text {max }}^{\text {sell }} .
\end{gathered}
$$

In addition, a binary variable, $\delta^{g}$, is considered and as per:

$$
\delta_{k}^{g}= \begin{cases}0 & \text { for selling energy } \\ 1 & \text { for buying energy }\end{cases}
$$

According to the mathematical formulation laid out above and different power flow directions in the given system, a global power balance equation is needed for any discrete sampling time $k$, which is defined as:

$$
\eta_{k}^{p v} P_{k}^{p v}+\eta_{k}^{d i s} P_{k}^{d i s}+P_{k}^{b u y}-\left(P_{k}^{s e l l}+P_{k}^{c h}+P_{k}^{l}\right)=0 .
$$

where $0<\eta^{c h}, \eta^{d i s}<1$, which balances the energy flow in/out in the BESS.

In this study, two electricity price functions are considered such as ToU which varies with time and feed-in-tariff which is assumed as constant, i.e., 0.1 AUD/kWh [14]. The ToU price function varies with three different price periods during a weekday: the shoulder-peak period from 07:00 to 13:59, and from 20:00 till 21:59; the high-peak hours from 14:00 to 19:59; the rest are off-peak hours. Weekends rates are shoulder and off-peak.

\section{Power Converter Efficiency}

In this paper, the case studied microgrid elements are interfaced with two power conversion converters, whose efficiency is an empirically modeled using a curve fitting technique, e.g. from quadratic interpolation [15].

$$
\eta=1-\frac{1}{R} \times\left(\alpha_{1}+\alpha_{2} \times R+\alpha_{3} \times R^{2}\right),
$$

where $R$ is the normalized input power of the converter which varies with time. the power conversion efficiency is calculated using (8) and applied on converters to solve the PMS.

Figure 2 illustrates, respectively, the power conversion efficiency characteristics of PV-connected and BESS-connected converters. It is observed that the efficiency of converter is lower when the input power to the converter is less than $15 \%$ of the nominal power.
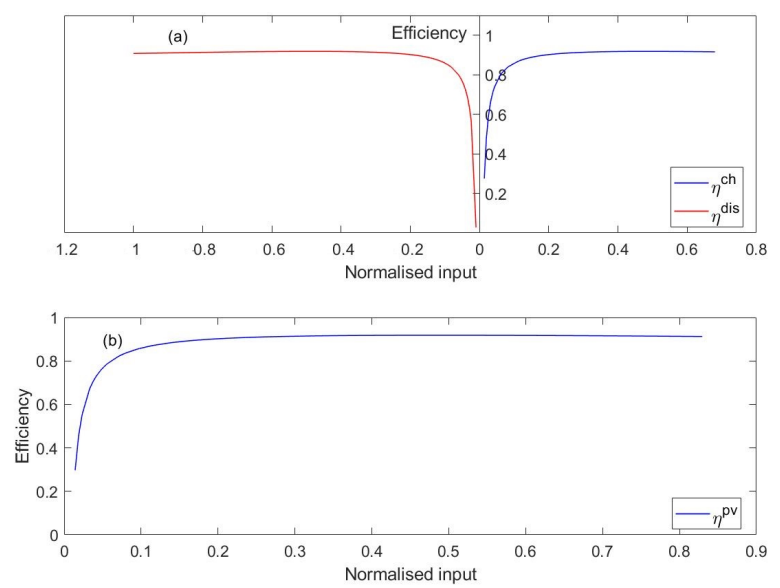

Fig. 2: Power converters efficiency plots. (a) BESS-connected bi-directional converter, (b) PV-connected converter.

\section{MILP-MPC FORMULATION}

Due to the dynamic nature of solar irradiation and power demand, this is a dynamic decision-making problem. Its objective is to minimize power consumption from the utility grid while satisfying operational constraints. Here, the power management problem can be viewed as a MILP optimization problem. The requirement of a real-time online control strategy has motivated us to use an MPC formulation.

As the problem is formulated in a MILP, which minimizes the linear function, $f^{T} u$, subject to the constraints, is typically defined as :

$$
\min _{u} f^{T} u \quad \text { subject to }\left\{\begin{array}{l}
\delta^{b} \text { and } \delta^{g} \text { are integers, } \\
A_{\text {ineq }} \cdot u \leq b_{\text {ineq }} \\
A_{e q} \cdot u=b_{\text {eq }} \\
l b \leq u \leq u b .
\end{array}\right.
$$

In this work, the objectives are to simultaneously minimize the energy consumption and maximize the energy selling at each instant $k$ over a week $(k \in\{0,1, \ldots, N-1\})$. To do this, it is required to find the optimal input variables, $\boldsymbol{u}_{k}^{o p t}$. Subsequently, The optimal economical problem can be defined as:

$$
J^{\text {opt }}=\min _{u} \sum_{k=0}^{N-1}\left(c_{k}^{\text {tou }} P_{k}^{\text {buy }} T_{s}-c_{k}^{f} P_{k}^{\text {sell }} T_{s}\right),
$$

where $u_{k}=\left[\begin{array}{llllll}P_{k}^{\text {dis }} & P_{k}^{\text {ch }} & P_{k}^{\text {buy }} & P_{k}^{\text {sell }} & \delta_{k}^{b} & \delta_{k}^{g}\end{array}\right]^{T}$. Here, The objective is to use predicted values of the electricity price, load demand and the available PV energy to find out the optimal consumption/selling at each time instant $k$. Consequently, we consider solving this optimal problem taking predictions into account over a desirable prediction horizon, i.e., $N_{p}<N$. Hence, the following optimal control problem can be solved at each instant $k$ as follows:

$$
\boldsymbol{u}_{k}^{\text {opt }}=\arg \min _{u} \sum_{j=0}^{N_{p}-1}\left(c_{j}^{\text {tou }} P_{j}^{\text {buy }} T_{s}-c_{j}^{f} P_{j}^{\text {sell }} T_{s}\right),
$$


subject to

$$
\begin{aligned}
& \delta_{j}^{b} P_{\min }^{d i s} \leq P_{j}^{d i s} \leq \delta_{j}^{b} P_{\max }^{d i s}, \\
& \left(1-\delta_{j}^{b}\right) P_{\min }^{c h} \leq P_{j}^{c h} \leq\left(1-\delta_{j}^{b}\right) P_{\max }^{c h}, \\
& 0 \leq P_{j}^{\text {buy }} \leq \delta_{j}^{g} P_{\text {max }}^{\text {buy }}, \\
& 0 \leq P_{j}^{\text {sell }} \leq\left(1-\delta_{j}^{g}\right) P_{\text {max }}^{\text {sell }}, \\
& 0 \leq \delta_{j}^{b} \leq 1, \\
& 0 \leq \delta_{j}^{g} \leq 1, \\
& S o C^{\text {min }} \leq \hat{S o C}_{j} \leq S o C^{\text {max }}, \\
& \hat{S o \hat{C}} C_{j+1}=\hat{S o ̂} C_{j}+T_{s} \eta_{k}^{c h} P_{j}^{c h}-T_{s} P_{j}^{d i s}, \\
& \eta_{k}^{p v} \hat{P} p v_{j}+\eta_{k}^{d i s} P_{j}^{d i s}+P_{j}^{\text {buy }}-\left(P_{j}^{s e l l}+P_{j}^{c h}+\hat{P}_{j}{ }_{j}\right)=0
\end{aligned}
$$

for all $j \in\left\{0,1, \ldots, N_{p}-1\right\}$, where the initial predictive values $\hat{S o} C_{0}=S o C_{k}, \hat{P^{p v}}{ }_{0}=P_{k}^{p v}, \hat{P}_{0}{ }_{0}=P_{k}^{l}, \hat{c}_{0}^{\text {tou }}=c_{k}^{\text {tou }}$ and $\hat{c}_{0}^{f}=c_{k}^{f}$ are known. So the optimal input sequence for the prediction horizon $N_{p}$ can be obtained as

$$
\boldsymbol{u}_{k}^{o p t}=\left[\begin{array}{llll}
\left(u_{0}^{o p t}\right)^{T} & \left(u_{1}^{o p t}\right)^{T} & \cdots & \left(u_{N_{p}-1}^{o p t}\right)^{T}
\end{array}\right]^{T} .
$$

Lastly, the decision variables to minimize the objective function at each sampling time $k$ is only the first element in $\boldsymbol{u}_{k}^{o p t}$, i.e.,

$$
u_{k}^{o p t}=u_{0}^{o p t},
$$

where the remaining optimal values in $\boldsymbol{u}_{k}^{\text {opt }}$, are discarded. The MILP optimization problem is repeated for the next sampling instant using new predicted values in order to attain a new optimal sequence $\boldsymbol{u}_{k+1}^{o p t}$. This process is known as receding horizon policy which is summarized in the proposed pseudocode Algorithm. Here, the prediction values of buying energy, selling energy, PV power, and load demand are obtained by the off-line prediction function $\operatorname{PRED}(\cdot)$ [16].

Remark 1. At each control interval, the MPC controller updates the plant model and evaluates each converter's efficiency by using current optimal input values. It is important to notice that $\eta^{p v}, \eta^{\text {ch }}$ and $\eta^{\text {dis }}$ are considered as persistent over prediction horizon, $N_{p}$.

The constraints in the optimization problem from (12) to (20) can be solved using a MILP technique and rewritten as the model (9), comprised of following matrices and vectors:

$$
\begin{gathered}
A_{\text {ineq }}=\left[\begin{array}{cccccc}
\boldsymbol{I} & \mathbf{0} & \mathbf{0} & \mathbf{0} & -P_{\max }^{\text {dis }} \boldsymbol{I} & \mathbf{0} \\
-\boldsymbol{I} & \mathbf{0} & \mathbf{0} & \mathbf{0} & P_{\min }^{\text {dis }} \boldsymbol{I} & \mathbf{0} \\
\mathbf{0} & \boldsymbol{I} & \mathbf{0} & \mathbf{0} & P_{\max }^{c h} \boldsymbol{I} & \mathbf{0} \\
\mathbf{0} & -\boldsymbol{I} & \mathbf{0} & \mathbf{0} & -P_{\min }^{\text {ch }} \boldsymbol{I} & \mathbf{0} \\
\mathbf{0} & \mathbf{0} & \boldsymbol{I} & \mathbf{0} & \mathbf{0} & -P_{\max }^{\text {buy } \boldsymbol{I}} \\
\mathbf{0} & \mathbf{0} & \mathbf{0} & \boldsymbol{I} & \mathbf{0} & P_{\max }^{\text {mall }} \boldsymbol{I} \\
T_{s} \boldsymbol{\phi} & -T_{s} \eta_{k}^{c h} \boldsymbol{\phi} & \mathbf{0} & \mathbf{0} & \mathbf{0} & \mathbf{0} \\
-T_{s} \boldsymbol{\phi} & T_{s} \eta_{k}^{c h} \boldsymbol{\phi} & \mathbf{0} & \mathbf{0} & \mathbf{0} & \mathbf{0}
\end{array}\right], \\
A_{e q}=\left[\begin{array}{llllll}
\eta_{k}^{d i s} \boldsymbol{I} & -\boldsymbol{I} & \boldsymbol{I} & -\boldsymbol{I} & \mathbf{0} & \mathbf{0}
\end{array}\right], \\
b_{e q}=\left[\left(P^{l}-\eta_{k}^{p v} P^{p v}\right)\right]^{T},
\end{gathered}
$$

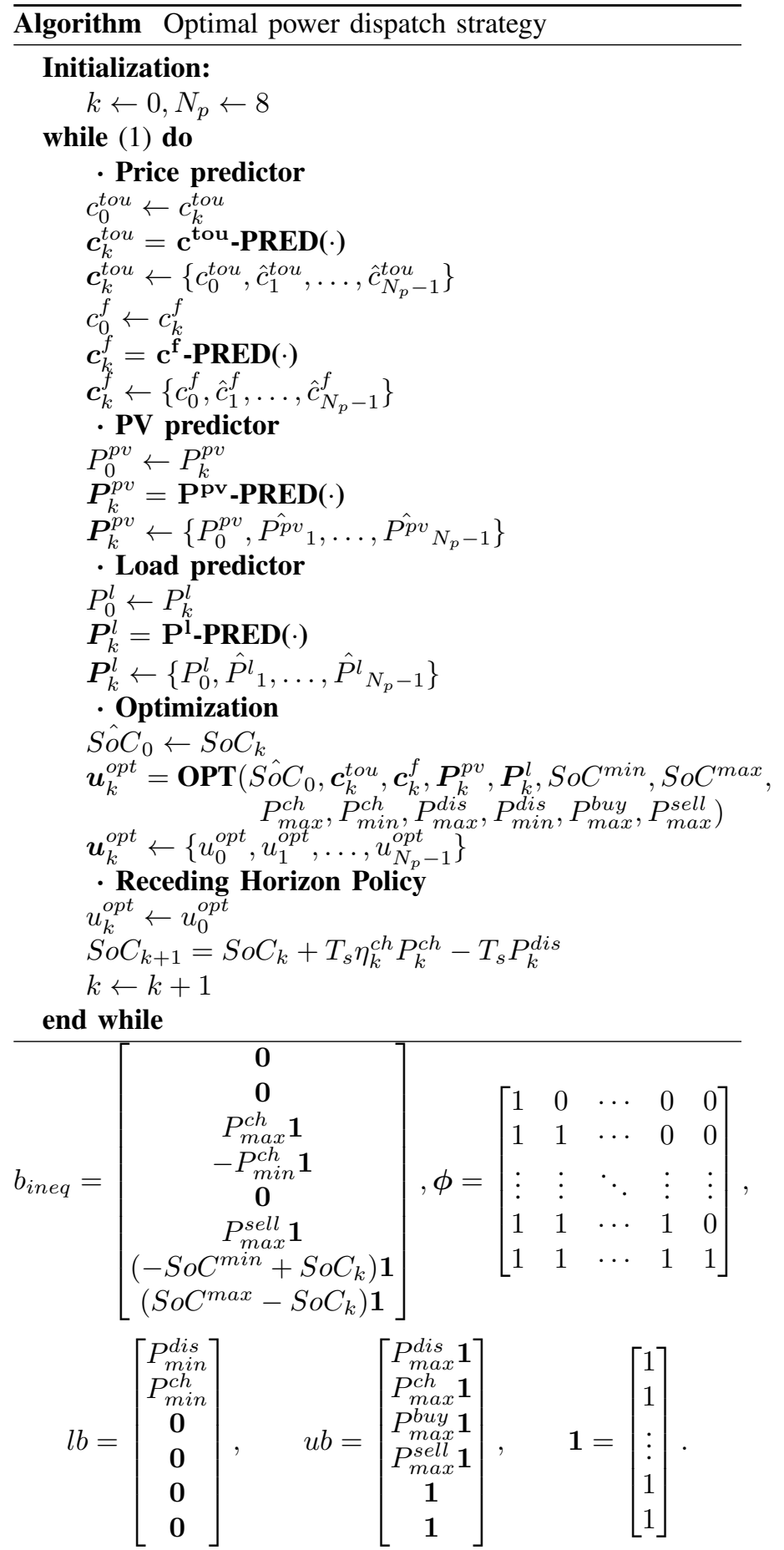

Remark 2. In this work, the MILP-MPC strategy depends on the predicted values of $P V$ generation, load demand and energy market price. It is important to notice that, we have not designed any actual predictor models in this paper. The function PRED(.) used here, gives off-line predictions by adding an error of $10 \%$ to the actual values. As matter of fact, the main objective is to attain an optimal battery schedule in order to minimize the electricity bill for a week.

\section{RESUlTS AND Discussions}

In this study, we considered the microgrid is in on-grid mode and consists of a PV system, BESS and power electronic 


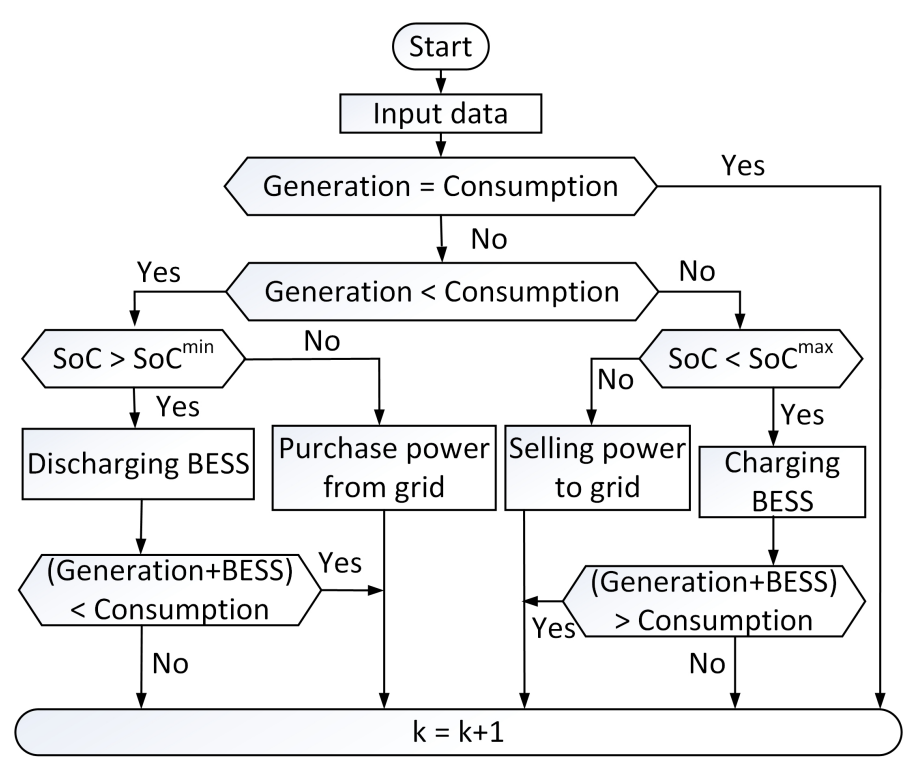

Fig. 3: Flowchart for the RBC algorithm.

converters interface. The proposed MILP-MPC strategy is tested with a real PV power data of a typical microgrid and real-time electricity market price data. The PV observation data has been taken from the PV system of an office building [17]. The nominal power of the PV system is $20 \mathrm{~kW}$ and the energy capacity of BESS is $100 \mathrm{kWh}\left(2 \times 50 \mathrm{kWh} \mathrm{Zn}-\mathrm{Br}_{2}\right.$ batteries). The values of the minimum and maximum rate of charging are $120 \mathrm{~W}$ and $17 \mathrm{~kW}$, whereas for discharging 20 $\mathrm{W}$ and $25 \mathrm{~kW}$ respectively. The storage energy available at the beginning of day is assumed to $20 \%$ of BESS capacity. In order to preserve the long life cycle, we considered the upper and lower limits for the BESS as 20\% (SoC $\left.{ }^{m i n}\right)$ and $80 \%$ $\left(S o C^{\max }\right)$ of its capacity. We choose a sampling time of 30 minutes such that one-week operational cycle consists of 336 discrete time intervals and simulations are performed for the period of a week.

The proposed MILP-MPC is compared with a rule-based control algorithm whose flowchart is shown in Fig. 3. Figure 4 depicts the power exchanged with BESS for the MILP-MPC and RBC strategies. The energy available at the beginning of the day is not sufficient to guarantee peak shaving for the rest of the day. Thus, with MILP-MPC, BESS absorbs the energy from PV and grid during low price periods, i.e., acting as a load to the system, and delivers electricity during high price periods, i.e., acting as a power generator. The energy stored in the battery using MILP-MPC and RBC strategies, is plotted in the Fig. 5. In both controlling strategies, it is important to notice that $S o C$ returns to the initial value at the end of the operational period for optimal power management for the next operational cycle which allows us to define absolute energy consumption cost for a week. The power exchange with the utility grid using MILP-MPC and RBC is illustrated in the Fig 6. As the energy selling price is constant and low as compared with the buying price, the proposed strategy preferred to charge the battery instead of selling the power to the utility grid when the excess PV power is available.
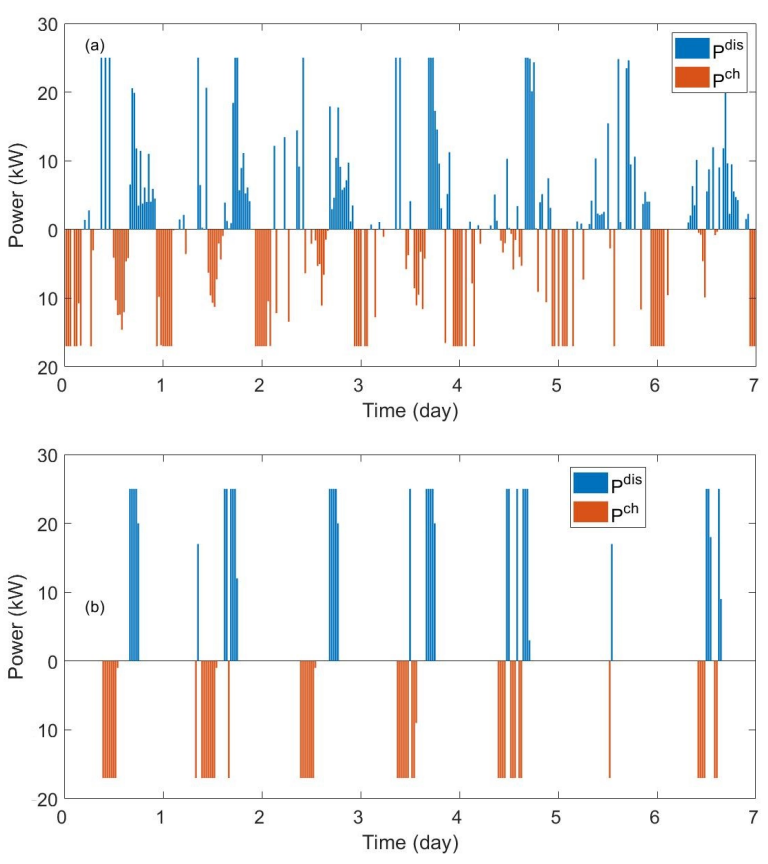

Fig. 4: Power exchanged with battery: (a) MILP-MPC, (b) RBC.
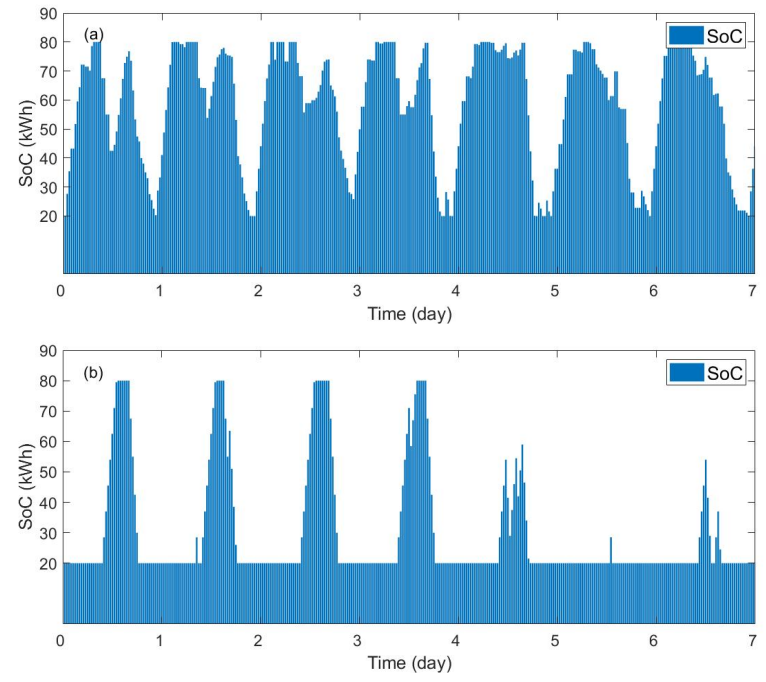

Fig. 5: Energy stored in the battery: (a) MILP-MPC, (b) RBC.

Due to the irregularity in PV power generation, more power is consumed from the utility grid in RBC strategy as compared with the proposed strategy. For a clear view, we plotted BESS and grid power profiles of the proposed method for the $2^{\text {nd }}$ day of the week in the Fig. 7. The electricity consumption cost per week MILP-MPC and RBC is detailed in Table I, indicates that the proposed method with variable efficiency results in a lower electricity bill with around $34.70 \%$ saving as compared to the RBC strategy. In this study, the simulation was repeated for MILP-MPC strategy with a fixed efficiency of $80 \%$, gives a $13.68 \%$ higher cost than the MILP-MPC strategy with variable efficiency. 

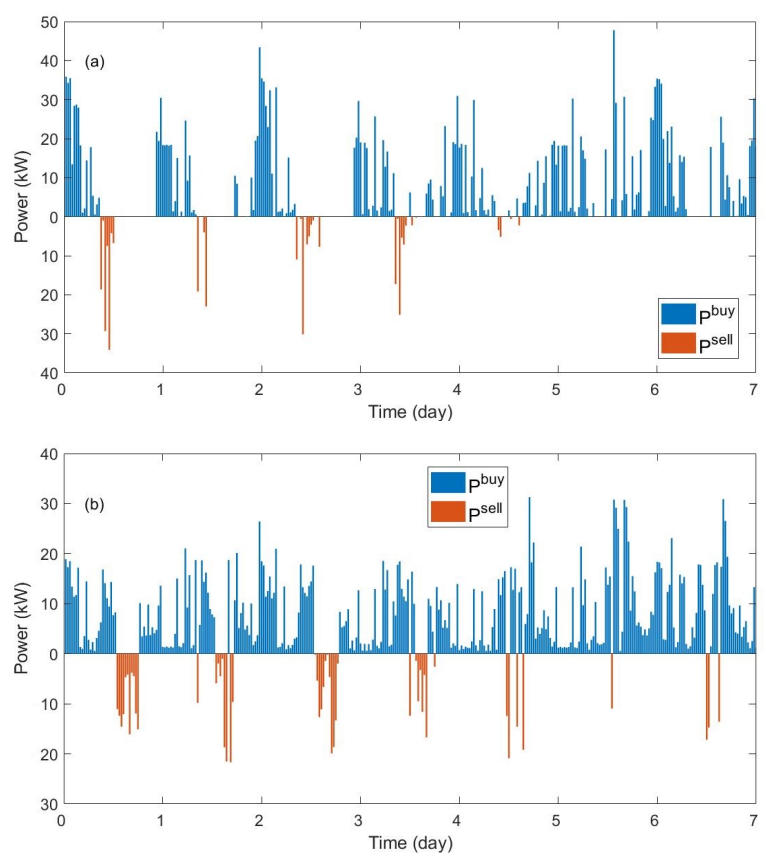

Fig. 6: Power exchanged with the utility grid: (a) MILP-MPC, (b) RBC.
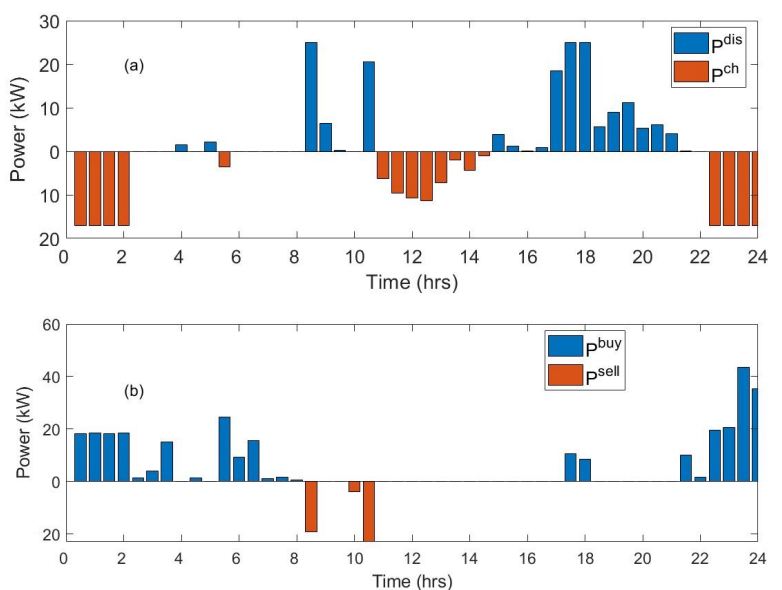

Fig. 7: MILP-MPC strategy for the $2^{\text {nd }}$ day: (a) Power exchanged with the battery, (b) Power exchanged with the utility grid.

TABLE I: Electricity bill for a week

\begin{tabular}{ccc}
\hline & & Electricity bill (AUD) \\
\hline \multicolumn{2}{c}{ RBC } & 239.15 \\
\hline \multirow{2}{*}{ MILP-MPC } & fixed $\eta$ & 181.38 \\
& variable $\eta$ & 156.56 \\
\hline
\end{tabular}

\section{CONCLUSION}

In this paper, a time-varying constrained MILP-MPC has been proposed for the PMS, that optimizes the energy cost of a commercial building energy system equipped with PV panels, BESS and grid connection. The proposed MILP-MPC takes into account the ToU electricity price function and dynamic power conversion losses which make the system more realistic. A case study of the energy management system of a real-world building is considered. The obtained numerical results indicate that the proposed MILP-MPC strategy with variable efficiency can store more energy to the battery with effective utilization of PV power generation. These together show the benefit in terms of saving the cost on electricity over the RBC strategy which is promising for the battery investment payback. Also, the proposed MILP-MPC with variable efficiency gives less power conversion losses as compared to MILP-MPC with fixed efficiency. Future work will focus on the implementation of the transactive power management and fair cost distribution among multiple house microgrids.

\section{REFERENCES}

[1] Y. Wang, X. Lin, and M. Pedram, "A near-optimal model-based control algorithm for households equipped with residential photovoltaic power generation and energy storage systems," IEEE Transactions on Sustainable Energy, vol. 7, no. 1, pp. 77-86, Jan 2016.

[2] X. Zhang, J. Bao, R. Wang, C. Zheng, and M. Skyllas-Kazacos, "Dissipativity based distributed economic model predictive control for residential microgrids with renewable energy generation and battery energy storage," Renewable Energy, vol. 100, pp. 18 - 34, 2017.

[3] I. Prodan and E. Zio, "A model predictive control framework for reliable microgrid energy management," International Journal of Electrical Power \& Energy Systems, vol. 61, pp. 399 - 409, 2014.

[4] M. J. Rana and M. A. Abido, "Energy management in de microgrid with energy storage and model predictive controlled ac \#8211;dc converter," IET Generation, Transmission Distribution, vol. 11, no. 15, pp. 36943702, 2017.

[5] M. El-Sharkh, A. Rahman, and M. Alam, "Evolutionary programmingbased methodology for economical output power from pem fuel cell for micro-grid application," Journal of Power Sources, vol. 139, no. 1, pp. $165-169,2005$.

[6] F. Mohamed and H. Koivo, "System modelling and online optimal management of microgrid using mesh adaptive direct search," International Journal Of Electrical Power and Energy Systems, vol. 32, no. 5, pp. 398-407, 2010.

[7] A. G. Tsikalakis and N. D. Hatziargyriou, "Centralized control for optimizing microgrids operation," IEEE Transactions on Energy Conversion, vol. 23, no. 1, pp. 241-248, March 2008.

[8] A. Parisio, E. Rikos, and L. Glielmo, "A model predictive control approach to microgrid operation optimization," IEEE Transactions on Control Systems Technology, vol. 22, no. 5, pp. 1813-1827, Sept 2014.

[9] A. Hooshmand, H. Malki, and J. Mohammadpour, "Power flow management of microgrid networks using model predictive control," Computers \& Mathematics with Applications, vol. 64, no. 5, pp. 869 - 876, 2012.

[10] M. Killian, M. Zauner, and M. Kozek, "Comprehensive smart home energy management system using mixed-integer quadratic-programming," Applied Energy, vol. 222, pp. 662 - 672, 2018.

[11] M. Khalid, R. P. Aguilera, A. V. Savkin, and V. G. Agelidis, "On maximizing profit of wind-battery supported power station based on wind power and energy price forecasting," Applied Energy, vol. 211, pp. $764-773,2018$.

[12] H. J. Bergveld, W. S. Kruijt, and P. H. L. Notten, Battery Management Systems: Design by Modeling. Springer Netherlands, 2013, vol. 1.

[13] R. K. Bonthu, H. Pham, R. P. Aguilera, and Q. P. Ha, "Minimization of building energy cost by optimally managing pv and battery energy storage systems," in 2017 20th International Conference on Electrical Machines and Systems (ICEMS), Aug 2017, pp. 1-6.

[14] Energy Australia, https://www.energyaustralia.com.au/home/help-andsupport/faqs/flexible-pricing, 2018, accessed on 09-Apr-2018.

[15] Y. Riffonneau, S. Bacha, F. Barruel, and S. Ploix, "Optimal power flow management for grid connected pv systems with batteries," IEEE Transactions on Sustainable Energy, vol. 2, no. 3, pp. 309-320, July 2011.

[16] M. Khalid, R. P. Aguilera, A. V. Savkin, and V. G. Agelidis, "A marketoriented wind power dispatch strategy using adaptive price thresholds and battery energy storage," Wind Energy, vol. 21, no. 4, pp. 242-254, 2018.

[17] University of Technology Sydney, "EIF Research Data Interface," https://eif-research.feit.uts.edu.au/graph/, 2017, accessed on 09-Aug2017. 